\title{
Supersolitons: Solitonic excitations in atomic soliton chains
}

\author{
David Novoa $^{1}$, Boris A. Malomed ${ }^{2}$, Humberto Michinel ${ }^{1}$, and Víctor M. Pérez-García ${ }^{3}$. \\ ${ }^{1}$ Área de Óptica, Facultade de Ciencias, Universidade de Vigo, As Lagoas s/n, Ourense, E-32004 Spain. \\ 2 Department of Physical Electronics, School of Electrical Engineering, \\ Faculty of Engineering, Tel Aviv University, Tel Aviv 69978, Israel \\ 3 Departamento de Matemáticas, E. T. S. I. Industriales, \\ and Instituto de Matemática Aplicada a la Ciencia y la Ingeniería, \\ Av. Camilo José Cela, 3, Universidad de Castilla-La Mancha, 13071 Ciudad Real, Spain.
}

\begin{abstract}
We show that, by appropriately tuning physically relevant interactions in two-component nonlinear Schrödinger equations, it is possible to achieve a regime with particle-like solitonic collisions. This allows us to construct an analogue of the Newton's cradle and also to create localized collective excitations in solitary-wave chains which are quasi-integrable solitons, i.e. supersolitons. We give a physical explanation of the phenomenon, support it with a perturbative analysis, and confirm our predictions by direct simulations.
\end{abstract}

PACS numbers: 05.45.Yv, 03.75.Lm

Introduction and model.- One of the most successful concepts of nonlinear science with applications to a great variety of physical contexts is that of solitons, i.e., selflocalized nonlinear waves, sustained by the balance between dispersion and nonlinearity. Many types of solitons have been studied, from the classical examples found in integrable models, such as the Korteweg - de Vries, sineGordon, Toda-lattice (TL), nonlinear Schrödinger, and other celebrated equations, and extending into the realm of realistic non-integrable nonlinear-wave models.

Solitons are usually expected to be robust against collisions, which is a trademark feature of integrable equations. A lot of activity has been directed at the study of soliton collisions and interactions in non-integrable systems. Recent advances include the analysis of chaotic scattering [1], the formation of soliton bound states and soliton clusters 2], and the studies of soliton collisions in vector systems [1, 3, 4], to name just a few.

While it is customary to speak of solitons as elastically colliding quasi-particles, most solitons, specifically in integrable systems, pass through each other, thus clearly featuring their wave nature. On the other hand, elastic collisions between classical particles lead to momentum exchange between them, and rebound, due to the nonpenetrability of classical particles.

In this paper we discuss a particular soliton collision scenario of physical relevance, where truly elastic particle-like soliton collisions can be achieved. We will show how this can be used to build a vector-soliton version of the Newton's cradle, and to build supersolitons, i.e. collective soliton-like excitations in arrays of solitary waves, leading to a remarkable conjunction of emergent phenomena: the former one representing the formation of robust soliton trains, and the latter effect implying the emergence of an effectively quasi-discrete soliton at a higher level of organization.

The basic model which allows to implement the abovementioned effects is based on the two-component (vecto- rial) nonlinear Schrödinger equation (NLSE), that arises in sundry contexts [5]. Its normalized form is

$$
i \frac{\partial u_{j}}{\partial t}=-\frac{1}{2} \frac{\partial u_{j}}{\partial x^{2}}+V(x) u_{j}+\sum_{k=1,2} g_{j k}\left|u_{k}\right|^{2} u_{j} .
$$

An important physical realization of this model is a multicomponent Bose-Einstein condensate (BEC), where $u_{j}$ are wave functions of two atomic states under the action of a strong transverse trap with frequency $\nu_{\perp}$ [6]. The variables $x$ and $t$ are measured, respectively, in units of $a_{0}=\sqrt{\hbar / m \nu_{\perp}}$ and $1 / \nu_{\perp}$, and $g_{i j} \equiv 2 a_{i j} / a_{0}$, with $a_{i j}$ the respective $s$-wave scattering lengths. The normalization integral for $u_{j}$ gives the number of atoms in the respective species, $\int_{-\infty}^{+\infty}\left|u_{j}\right|^{2} d^{3} x=\mathcal{N}_{j}$. Solitons in BECs have been created experimentally [7] and their interactions studied theoretically in many papers (see e.g. [8, 9, 10]).

Particle-like elastic collisions and the solitonic Newton's cradle.- We will consider Eqs. (11) with intracomponent attraction $\left(g_{11}, g_{22}<0\right)$ and inter-component repulsion $\left(g_{12}, g_{21}>0\right)$. To fix ideas we will choose $g_{11}=g_{22}=-g_{12}=-g_{21}(=1$ without loss of generality $)$ for which case the coupled NLSE are not integrable [11]. In this situation the solitons, that may be formed in both components independently, interact incoherently with a repulsive force. The basic physical feature underlying our analysis is that the dynamics of those solitons may be similar to that of elastic beads. We will explore the cases of harmonic longitudinal confinement $V(x)=\Omega^{2} x^{2} / 2$, and ring-shaped configurations 12 .

Let us consider soliton trains built as follows:

$$
\begin{aligned}
& u_{1}(x, 0)=\sum_{n=1, \ldots, N}(-1)^{n} \operatorname{sech}\left(x-\xi_{n}\right) \exp \left(i x v_{n}\right)(2 \mathrm{a}) \\
& u_{2}(x, 0)=\sum_{n=1, \ldots, N}(-1)^{n} \operatorname{sech}\left(x-\zeta_{n}\right) \exp \left(i x w_{n}\right)(2 \mathrm{~b})
\end{aligned}
$$

with alternation of the soliton species in the train, i.e.,

$$
\ldots \xi_{n-1}<\zeta_{n-1}<\xi_{n}<\zeta_{n}<\xi_{n+1}<\zeta_{n+1}<\ldots,
$$


$v_{n}$ and $w_{n}$ being initial velocities of the solitons. In Fig. 1. where the trap is absent, panel (a) displays a single collision event $(N=1)$. It is noteworthy that, because of the repulsive inter-component interaction, the incident soliton (in field $u_{1}$ ) transfers all of its momentum to the initially static soliton (in $u_{2}$ ), in full compliance with the behavior of elastic particles and contrary to the typical behavior of nontopological solitons in integrable systems. The dynamics of a train of eight alternating solitons in a ring configuration demonstrates the periodic transfer of the momentum through the train, see Fig $1(\mathrm{~b})$.
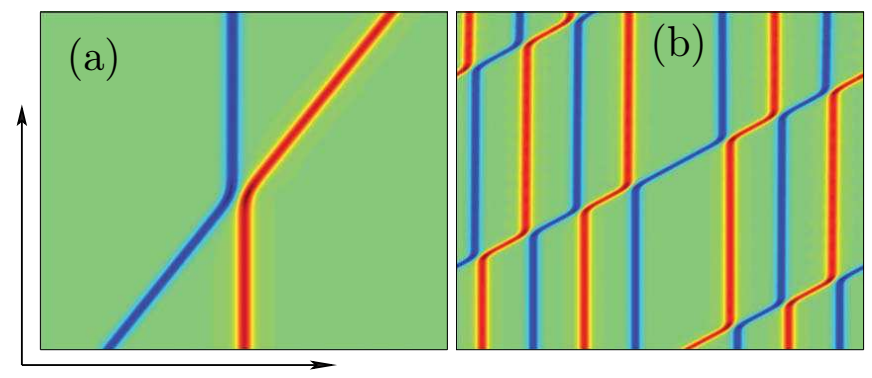

FIG. 1: [Color online] Pseudocolor plots of $\left|u_{1}(x, t)\right|^{2}$ (blue) and $\left|u_{2}(x, t)\right|^{2}$ (red) show the time evolution of initially well separated soliton trains built as per Eq. (2). The horizontal (vertical) axis of each plot is spatial adimensional variable $x$ (adimensional time $t$ ). (a) Collision of two solitons $(N=1)$ for $\xi_{1}=-20, \zeta_{1}=0, v_{1}=0.4, w_{1}=0, x \in[-30,30]$ and $t \in[0,100]$. (b) Multisoliton collisions in a ring for $N=4$, $\zeta_{j}=-35+20 j, \xi_{j}=-25+20 j, x \in[-40,40]$ and $t \in[0,250]$. Initial velocities are all zero except for $w_{3}=0.5$.

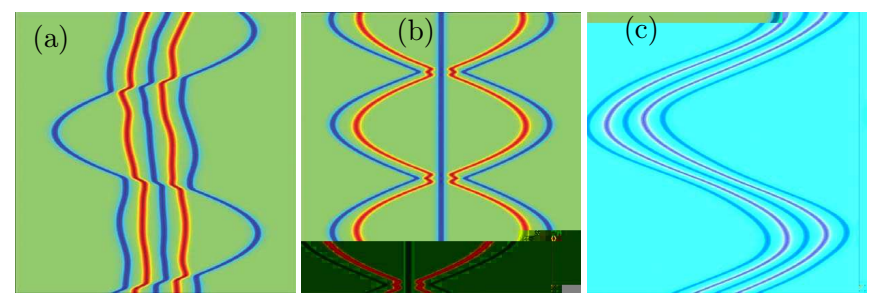

FIG. 2: (Color online) Same as Fig. 1 for a Newton's cradle built up of five solitons with an external potential with $\Omega^{2}=$ $6 \cdot 10^{-5}$. In all subplots $t_{\max }=1000, x \in[-40,40]$, and the initial separation between solitons is $\xi_{n}-\zeta_{n}=6$. Different oscillation modes are excited by applying an initial velocity, of size 0.2, to: (a) the top-left soliton, (b) two ultimate solitons on both sides, with opposite velocities, and (c) all solitons.

By adding the parabolic trapping potential we urge the solitons to oscillate around the equilibrium position. In the quantum interpretation of our model this setup would provide a way to construct a quantum Newton's cradle with atomic solitons, as illustrated in Fig. 2, Unlike other settings explored in BEC [13], the cradle configuration does not require a lattice potential to create effective particles, which are here created by purely nonlinear interactions.

The Toda-lattice limit: supersolitons - We now consider two alternating chains of solitons set along a ring. Within the effective-particle approach, we assume that all solitons, which have identical amplitudes in each component, $\eta$ (for $u_{1}$ ) and $\theta$ (for $u_{2}$ ), behave like rigid particles and thus do not suffer conspicuous deformation, i.e., each soliton may be approximated by

$$
\begin{aligned}
& u_{1}^{(n)}(x, t)=\eta \operatorname{sech}\left[\eta\left(x-\xi_{n}\right)\right] e^{i \dot{\xi}_{n} x+\frac{1}{2} \int\left(\eta^{2}-\dot{\xi}_{n}^{2}\right) d t},(4 \mathrm{a}) \\
& u_{2}^{(n)}(x, t)=\theta \operatorname{sech}\left[\theta\left(x-\zeta_{n}\right)\right] e^{i \dot{\zeta}_{n} x+\frac{1}{2} \int\left(\theta^{2}-\dot{\zeta}_{n}^{2}\right) d t},(4 \mathrm{~b})
\end{aligned}
$$

with the initial positions arranged as per Eq. (3).

A straightforward analysis based on the perturbation theory for solitons [14] yields the following system of equations of motion for the soliton coordinates:

$$
\begin{aligned}
\ddot{\xi}_{n}= & -\Omega^{2} \xi_{n}+8 \theta\left[\eta^{2}\left(e^{-2 \eta\left(\xi_{n}-\zeta_{n-1}\right)}-e^{-2 \eta\left(\zeta_{n}-\xi_{n}\right)}\right)\right. \\
& \left.+\theta^{2}\left(e^{-2 \theta\left(\xi_{n}-\zeta_{n-1}\right)}-e^{-2 \theta\left(\zeta_{n}-\xi_{n}\right)}\right)\right], \\
\ddot{\zeta}_{n}= & -\Omega^{2} \zeta_{n}+8 \eta\left[\eta^{2}\left(e^{-2 \eta\left(\zeta_{n}-\xi_{n}\right)}-e^{2 \eta\left(\xi_{n+1}-\zeta_{n}\right)}\right)\right. \\
& \left.+\theta^{2}\left(e^{-2 \theta\left(\zeta_{n}-\xi_{n}\right)}-e^{-2 \theta\left(\xi_{n+1}-\zeta_{n}\right)}\right)\right] .
\end{aligned}
$$

These equations are derived under the assumption that adjacent solitons are well separated, i.e., $(\eta, \theta)\left(\xi_{n}-\zeta_{n-1}\right),(\eta, \theta)\left(\zeta_{n}-\xi_{n}\right) \gg 1$, although a strong inequality is not really necessary here. Similar ideas have been used to derive equations for the interaction of other elementary nonlinear structures, which gives rise to different equations at a higher level of organization [3, 10, 15]. Equations (5) with $\Omega=0$ reduce to the so-called diatomic TL, which is not integrable, although some solutions are known.

With $\eta=\theta$ and $\Omega=0$ in Eqs. (5) and defining $q_{2 n}(t)=$ $2 \eta \xi_{n}(t), q_{2 n+1}(t)=2 \eta \zeta_{n}(t)$ and $\alpha=32 \eta^{4}$, we arrive at the integrable TL model [16],

$$
\ddot{q}_{n}=\alpha\left[e^{-\left(q_{n}-q_{n-1}\right)}-e^{-\left(q_{n+1}-q_{n}\right)}\right] .
$$

This model describes a dynamical lattice with the exponential potential of the nearest-neighbor interaction. However, potentials of the interaction between adjacent atoms in real condensed-matter systems are never exponential, being closer to those of nonlinear anharmonic oscillators. This is why the only experimental realization of the integrable TL was realized in electric transmission lines [18], that may be readily designed in exact correspondence to Eqs. (6). Our analysis suggests a possibility to create Toda solitons, of both mono- and diatomic types, as excitations in interwoven arrays of multicomponent NLSE solitary waves.

We name these excitations supersolitons since they occur on top of an array of "elementary" solitons, and are 
expected to be as robust as solitons in integrable models. The same name was previously applied to solitons in supersymmetric models [17], and, in a completely different context, to localized topological collective excitations in chains of fluxons trapped in periodically inhomogeneous Josephson junctions and in layered superconducting structures [15]. The appearance of TL supersolitons represents a remarkable phenomenon at a higher-organization level, using, as building blocks, solitary waves of the multicomponent NLSE, i.e. a strongly nonintegrable model.

In the monoatomic lattice, Eq. (6) has an obvious equilibrium solution with $q_{n}=L /(2 N)$, where $N$ is the number of solitons in each subchain, and $L$ the total length of the system. For small perturbations with frequency $\omega$ and wavenumber $k$ around this configuration, the dispersion relation is $\omega^{2}=128 \eta^{4} e^{-\eta L / N} \sin ^{2}(k / 2)$. With respect to the quantization imposed by the boundary conditions for the ring-shaped soliton chain, $k=\pi m / N$, $m=0, \pm 1, \pm 2, \ldots$, this yields

$$
\left|\omega_{m}\right|=8 \sqrt{2} \eta^{2} \exp (-\eta L / 2 N)|\sin (\pi m /(2 N))| \text {. }
$$

If a wave in the lattice is excited by kicking one soliton and lending it velocity $v$, the wave will hit solitons with period $T=L /(2 N v)$, which corresponds to an effective excitation frequency $\omega_{\text {exc }} \equiv 2 \pi / T=4 \pi N v / L$. Thus, resonant excitations may be expected under the condition $P \omega_{\text {exc }}=Q\left|\omega_{m}\right|$, or, in other words, at values of the kick velocity belonging to the following resonant spectrum,

$$
\left|v_{m}^{(P, Q)}\right|=\frac{Q}{P} \cdot \frac{2 \sqrt{2} L \eta^{2}}{\pi N} e^{-\eta L / 2 N}|\sin (\pi m /(2 N))|,
$$

where integers $Q$ and $P$ stand for the order of the resonance and subresonance $(P=Q=1$ correspond to the fundamental resonance). Another interpretation of this resonance condition (cf. Ref. [19]) is that the kick velocity coincides with the phase velocity of linear waves.

Numerical studies of supersolitons - To verify our predictions based on Eq. (6), we have performed numerical simulations of Eq. (1). First, in Fig. 3 we have generated a single supersoliton by kicking one of the most external solitons in one of the components. Since this excitation does not correspond exactly to a supersoliton we also obtain a small ammount of radiation which is seen as small remnant oscillations of the individual solitary waves. Apart from this efect due to the excitation procedure, the propagation of the supersoliton is perfect as seen both in the amplitude [Fig [3(a)] and pseudocolor [Fig. 3(b)] plots. Another effect seen in Fig. 3(a) and not considered in our model is the small compression of the individual solitary waves when they are hit by the supersoliton (the model assumes equal amplitude individual solitary waves). However this small effect does not affect our conclusions and can be minimized by considering smaller energy collisions (i.e. incident speeds).
Fig. 4 shows the collisional behavior for head on collisions of equal speed solitons [Fig. 4(a)] and the overtaking of a slow supersoliton by a faster one [Fig. 4(b)]. In both cases the supersolitonic excitations behave as true solitons, what it is justified by the integrability of our simple model given by Eqs. (6). We want to emphasize again that these behaviors, typical of integrable systems, arise on top of a strongly nonintegrable model.

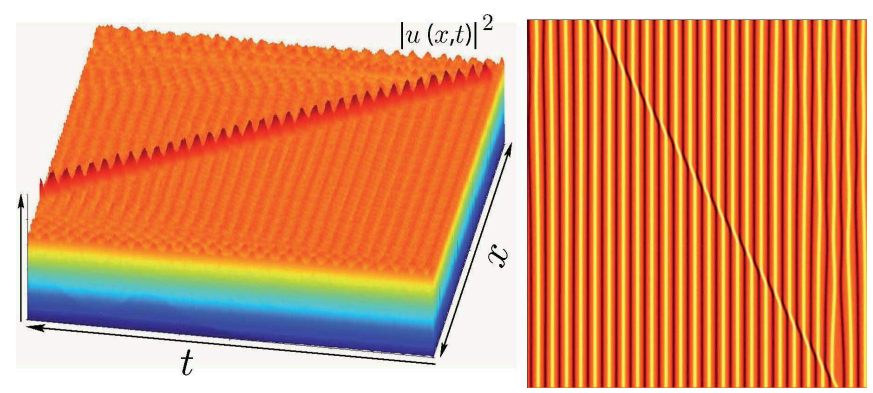

FIG. 3: (Color online) Excitation of a supersoliton on a soliton chain given by Eq. (2-3) with $N=24$ localized solitons in each component, by kicking the $j=22$ soliton with velocity $w_{22}=-0.5$. Individual solitons have initial amplitudes $\eta=\theta=1$ and the intersoliton distance is five units leading to a total ring length of 240. Left: Amplitude plot of $|u(x, t)|^{2}=\left|u_{1}(x, t)\right|^{2}+\left|u_{2}(x, t)\right|^{2}$. Right: Pseudocolor plot showing $\left|u_{1}(x, t)\right|^{2}$ (yellow) and $\left|u_{2}(x, t)\right|^{2}$ (red). The range of adimensional times spanned in both plots is $t \in[0,250]$.

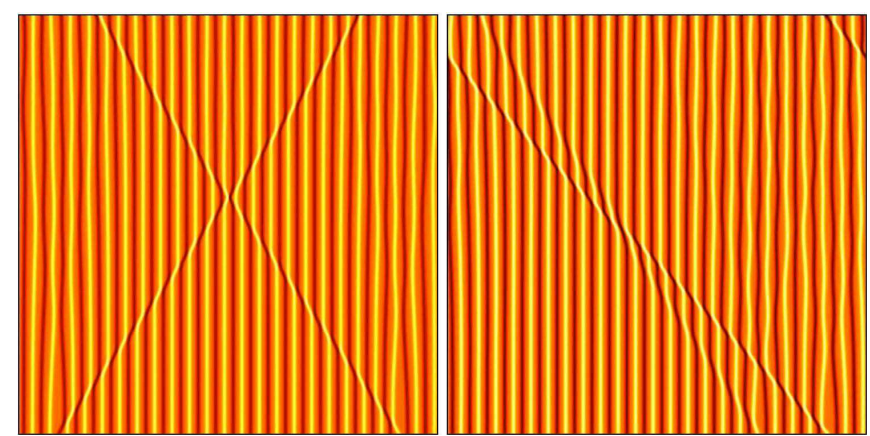

FIG. 4: (Color online) Examples of supersoliton collisions. The initial configuration is as in Fig. 3 but now two supersolitons are excited. Left: Head-on collision induced with excitation parameters $v_{3}=0.5$ and $w_{22}=-0.5$. The range of adimensional times spanned is $t \in[0,250]$. Right: Soliton overtaking excited with initial velocities $v_{23}=-0.6, w_{19}=-0.3$. The range of adimensional times spanned is $t \in[0,350]$.

Can scalar models support supersolitons?- Soliton collisions in the framework of scalar NLSEs have been studied in various contexts, and equations similar to Eqs. (5) have been derived using different approaches [3, 10], leading to the so-called complex TL. Despite the formal similarities, the ensuing dynamics is not robust, and solitonic solutions turn out to be unstable because of the 
phase dependence of the interactions. An example is displayed in Fig. 5(a), which shows that the phase shifts induced by the initial kick velocity lead to an unstable dynamics of the single-component chain, whereas in its alternating two-component counterpart the system does not display any instability, as shown in Fig. [5(b); in particular, the configuration shown in Fig. 5(b) periodically recovers its shape. Thus, the vectorial system with incoherent interactions is free of the instability of soliton chains in single-component models with coherent (phasedependent) interactions [20].

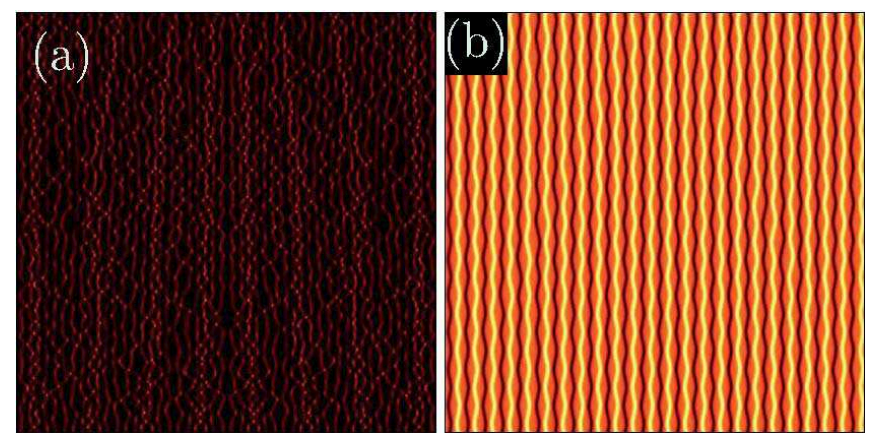

FIG. 5: (Color online) Time evolution for $0<t<500$ of matter-wave soliton trains with a small collective excitation in the case of one (a) and two (b) species, which are described, respectively, by the scalar and coupled NLSEs (black and white colors). Adjacent solitons are given opposed input velocities, $\chi= \pm 0.1$ in (a), and $v_{j}=0.1, w_{j}=-0.1$ in (b). Other parameters are as in Fig. [3]

Experimental realization.- The creation of TL supersolitons in BECs would depend on the use Feshbach resonance techniques to get an atomic mixture with attractive intra-species and repulsive inter-species interactions. Atomic mixtures with controllable interspecies interactions have already been reported in Ref. [21]. Our initial state of alternating solitons may be created by the modulational instability and segregation from an initially stable two-component mixture [22].

Conclusions.- We have explored a physical model based on the vectorial NLSE, in which hard-particle-like (bouncing) elastic collisions between solitons belonging to different species are possible. These interactions allow building an analogue of the Newton's cradle using solitary waves, and to supersolitons in a chain of alternating solitons. The existence of these robust localized collective excitations on top of arrays of nonintegrable solitons represents a remarkable emergent phenomenon.

Acknowledgements.- This work has been partially supported by grants FIS2006-04190, FIS2004-02466, FIS2007-29090-E (Ministerio de Educación y Ciencia, Spain), PGIDIT04TIC383001PR (Xunta de Galicia) and PCI-08-0093 (Junta de Comunidades de Castilla-La Mancha, Spain). D.N. acknowledges a grant from Consellería de Educación (Xunta de Galicia).
[1] J. K. Yang and Y. Tan, Phys. Rev. Lett. 85, 3624 (2000); V. Dmitriev et al., Phys. Rev. E 68, 056603 (2003); R. H. Goodman and R. Haberman, Phys. Rev. Lett. 98, 104103 (2007).

[2] J. K. Yang et al., Phys. Rev. Lett. 94, 113902 (2005); M. Stratmann, T. Pagel, and F. Mitschke, ibid. 95, 143902 (2005); Y. Leitner and B. A. Malomed, Phys. Rev. E 71, 057601 (2005); C. Rotschild et al., Nature Physics 2, 769 (2006); D. Buccoliero et al., Phys. Rev. Lett. 98, 053901 (2007); J. Belmonte-Beitia et al.,ibid. 98, 064102 (2007).

[3] V. S. Gerdjikov, and I. M. Uzunov, Physica D 152 (2001) 355.

[4] T. Kanna et al., Phys. Rev. E 73, 026604 (2006); T.-S. Ku et al., Phys. Rev. Lett. 94, 063904 (2005).

[5] Y. Kivshar, G. P. Agrawal, Optical Solitons: From fibers to Photonic crystals, Academic Press (2003); L. P. Pitaevskii and S. Stringari, Bose-Einstein Condensation, Oxford University Press, Oxford (2003).

[6] V. M. Pérez-García, H. Michinel, and H. Herrero, Phys. Rev. A 57, 3837 (1998).

[7] S. Burger et al., Phys. Rev. Lett. 83, 5198 (1999); G. B. Partridge, A .G. Truscott, and R. G. Hulet, Nature 417, 150 (2002); L. Khaykovich et al., Science 296, 1290 (2002); B. Eiermann et al., Phys. Rev. Lett. 92, 230401 (2004); S. L. Cornish, S. T. Thompson, and C. E. Wieman, Phys. Rev. Lett. 96, 170401 (2006).

[8] L. D. Carr and J. Brand, Phys. Rev. Lett. 92, 040401 (2004); V. M. Pérez-García and J. Belmonte-Beitia, Phys. Rev. A 72, 033620 (2005); J. Babarro, et al., ibid., A 71, 043608 (2005); L. Khaykovich and B. A. Malomed, ibid. 74, 023607 (2006); T. Lin et al., Nonlinearity 19, 2755 (2006).

[9] V. S. Gerdjikov, B. B. Baizakov, and M. Salerno, Theor. Math. Phys., 144, 1138 (2005).

[10] A. D. Martin, C. S. Adams, and S. A. Gardiner, Phys. Rev. Lett. 98, 020402 (2007).

[11] V. E. Zakharov and E. I. Schulman, Physica D 4, 270 (1982).

[12] S. Gupta et al., Phys. Rev. Lett. 95, 143201 (2005); C. Ryu et al., Phys. Rev. Lett. 99, 260401 (2007).

[13] T. Kinoshita, T. Wenger and D. S. Weiss, Nature 440, 900 (2006).

[14] Yu. S. Kivshar and B. A. Malomed, Rev. Mod. Phys. 61, 763 (1989).

[15] A. V. Ustinov, Phys. Lett A 136, 155 (1989); B. A. Malomed, Phys. Rev. B 41, 2616 (1990); Y. S. Kivshar, T. K. Soboleva, Phys. Rev. B, 42, 2655(R) (1990).

[16] M. Toda, Theory of Nonlinear Lattices, (Springer-Verlag, New York 1989).

[17] Yu. I. Manin, A. Radul, Commun. Math. Phys. 98, 65 (1985); A. S. Carstea, Nonlinearity, 13, 1645 (2000); Y. Ya-Xuan, Commun. Theor. Phys. 49, 685 (2008).

[18] See e.g. P. Marquie, J. M. Bilbault, and M. Remoissenet, Phys. Rev. E 51, 6127 (1995) and references therein.

[19] A. V. Ustinov, M. Cirillo, and B. A. Malomed, Phys. Rev. B 47, 8357 (1993).

[20] T. Gallay and M. Haragus, J. Dyn. Diff. Equat.19, 825 (2007), and references therein.

[21] G. Thalhammer, et al., arxiv:0803.2763v1

[22] K. Kasamatsu and M. Tsubota, Phys. Rev. Lett. 93, 100402 (2004); Phys. Rev. A 74, 013617 (2006). 